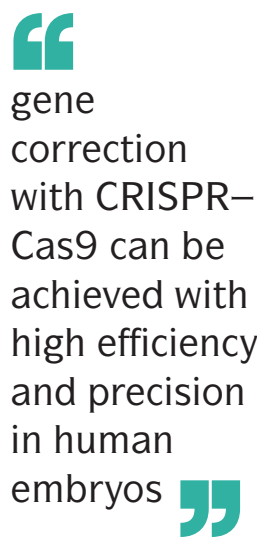

BIOTECHNOLOGY

\title{
CRISPR-Cas becoming more human
}

In the past few years, the CRISPR-Cas technology has been developed to precisely edit genomic DNA; so far, these approaches have been used mainly in cultured cells or in animal embryo models, but only a few studies have reported their use in human embryos. Ma et al. now report that CRISPR-Cas9 can efficiently and safely correct a pathogenic heterozygous mutation in human embryos.

Mutations in MYBPC3 account for $\sim 40 \%$ of all genetic defects causing hypertrophic cardiomyopathy, which is a common cause of heart failure in otherwise healthy individuals. Many of these autosomal mutations are dominant, and inheritance of a single copy results in pathological symptoms. $\mathrm{Ma}$ et al. set out to specifically correct a heterozygous dominant 4 bp deletion in MYBPC3 (MYBPC $\left.3^{\triangle \mathrm{GAGT}}\right)$, using a single guide RNA (sgRNA)Cas 9 construct that was designed to target this specific deletion.

CRISPR-Cas9 recognizes specific genomic sequences and induces DNA double-strand breaks (DSBs), which can be repaired by a non-homologous end-joining (NHEJ) repair pathway or by homology-directed repair (HDR). Repair by NHEJ is error prone, and is unsuitable for correcting mutations for therapeutic purposes as it introduces additional mutations.

As, unlike NHEJ, HDR can restore the wild-type sequence, the authors first evaluated HDR efficiency in 54 human embryos that were obtained by fertilizing healthy donor oocytes with sperm donated by an adult patient carrying the $M Y B P C 3^{\triangle \mathrm{GAGT}}$ heterozygous mutation.
The sgRNA and the Cas9 protein were injected into S-phase zygotes postfertilization, and sequencing of all blastomeres in each embryo revealed that 36 embryos (66.7\%) were uniformly homozygous for the wild-type allele, whereas, for the remaining 18 embryos, 5 were uniformly heterozygous (not targeted by CRISPR-Cas9) and 13 were mosaics containing a mixture of homozygous wild-type and heterozygous mutant blastomeres that contained both $M Y B P C 3^{\triangle \mathrm{GAGT}}$ alleles and alleles resulting from NHEJ. These results indicated that CRISPR-Cas9 can efficiently target mutations in human embryos, but, for clinical applications, mosaicism must be entirely avoided, as a single mutant blastomere could give rise to a mixture of healthy and diseased cells as adult tissues develop.

The authors reasoned that mosaicism could result either from CRISPR-Cas9 being active after zygotic division or from DNA being targeted after DNA replication (producing two paternal mutant alleles). Both of these possible problems were overcome by mixing sperm and CRISPR-Cas9 components and co-injecting them into oocytes arrested in meiosis. Of the 58 injected embryos, 42 (72.4\%) were homozygous for the wild-type allele, and the level of mosaicism was very low (1 in 42 embryos), presumably as a result of gene editing occurring before the first zygotic division. In the remaining 16 embryos, $M Y B P C 3^{\triangle \mathrm{GAGT}}$ was repaired by NHEJ, resulting in different mutant alleles. Together, this shows that CRISPRCas9 editing is extremely efficient in M-phase fertilized oocytes, targeting all mutant sequences (unlike in patient-derived induced pluripotent stem cells (iPSCs), in which targeting efficiency was $27.9 \%$ ). Interestingly, the authors found that HDR occurs using the endogenous maternal allele as a template for repair, rather than exogenously supplied oligonucleotides as happens in cultured cells, suggesting that embryos and germ cells employ different mechanisms for DNA repair.

Importantly, no off-target mutations (which can result from editing components binding to sequences with high similarity elsewhere in the genome) were detected in the modified embryos, suggesting that this particular sgRNA is highly specific. Whether off-target mutations are indeed absent is difficult to prove, but the results from Ma et al. are highly promising. Moreover, when cultured to the blastocyst stage, embryos following CRISPR-Cas9 editing did not show any developmental defects or cytological abnormalities, indicating that they can grow normally.

This study suggests that gene correction with CRISPR-Cas9 can be achieved with high efficiency and precision in human embryos. However, before the technique can be considered a safe therapy for inherited diseases, it requires further optimization to entirely abolish embryo mosaicism and the absence of off-target mutations needs to be thoroughly validated. The findings will undoubtedly heat up ethical debates about editing embryos and the germline.

Kim Baumann, Chief Editor, Nature Reviews Molecular Cell Biology This article is modified from the original in Nat. Rev. Mol. Cell Bio. (http://dx.doi.org/10.1038/nrm.2017.84)

ORIGINAL ARTICLE Ma, H. et al. Correction of a pathogenic gene mutation in human embryos. Nature http://dx.doi.org/10.1038/nature23305 (2017)

FURTHER READING Fellmann, C. et al. Cornerstones of CRISPR-Cas in drug discovery and therapy. Nat. Rev. Drug Discov. 16, 89-100 (2017) 